\title{
EDITORIAL
}

\section{'Educational symposium on long-term results of large prospective clinical trials for childhood acute lymphoblastic leukemia (1985-2000)'}

Leukemia (2010) 24, 253-254; doi:10.1038/leu.2009.276

\section{Introduction}

There has been steady improvement in outcome for children and adolescents with acute lymphoblastic leukemia (ALL) in the past 40 years. In this decade, we can expect that at least $80 \%$ of patients with ALL will be cured with their initial course of chemotherapy and an additional $5-8 \%$ of patients can be cured after retrieval therapy for relapse. It is truly remarkable that 85$90 \%$ of children are cured today of a disease that was universally fatal within weeks to months in the early 1960s. With marked reduction in the use of cranial radiation and risk-directed therapy that stratifies low-risk patients to receive less toxic therapy, we can also expect a significant improvement of the quality of life of the survivors. Recognizing the necessity of international collaboration to further improve the cure rates and the quality of life of patients, major childhood ALL study groups agreed to make a concerted effort to identify important treatment strategies. As a result, in this issue of Leukemia, an international representation of study groups will report and compare the long-term results of patients treated between 1985 and 2000.

The first series of papers coordinated in a similar fashion was published in Leukemia in 2000 (Vol. 14). Now, 10 years later, these groups have again agreed to use the same or similar criteria to present their results - in addition to the stratification criteria originally designed for their studies. Only through this large, unique and essential exercise could one compare longterm outcome results among various clinical trials to identify effective treatment strategies for specific subsets of childhood ALL. Disease recurrence and treatment failure have been carefully analyzed to account for the various causes for lack of success. Some groups (for example, the Japanese Childhood Cancer and Leukemia Study Group (JCCLSG)) have even provided details of late adverse effects. Overall, 77 studies from 15 study groups have been reanalyzed to present updated longterm outcome results from a total of 52891 patients (Table 1). To coincide with the protocol enrollment period, some groups elected to include patients treated since 1980 and others included those treated as recently as 2007. It should be pointed out, however, that not all studies presented here are population based, and thus, the results reported here do not necessarily reflect the overall treatment quality and cure rates achieved in respective countries and health systems.

\section{A tribute to Haig Riehm}

During the workshop and educational symposium, there was a consensus to dedicate this monogram to Professor Hansjörg (Haig) Riehm whose monumental contributions had a major role in making childhood ALL a highly curable disease. In the late 1960s and early 1970s, the first cures of childhood ALL were produced by treatment regimens that consisted of a three-drug remission induction with vincristine, prednisone and asparaginase, a central nervous system intensification phase consisting of weekly intrathecal methotrexate, cranial radiation and daily oral mercaptopurine and continuation therapy with monthly pulses of prednisone and vincristine in conjunction with daily oral mercaptopurine and weekly oral methotrexate. However, the majority of patients treated with these regimens eventually relapsed and died from their disease.

At the same time, Dr Riehm, a young pediatric oncologist in Germany, developed a new regimen for treating childhood ALL based on the Goldie Goldman and the Norton Simon hypotheses. He first reasoned that it was critical to use multiple agents early in treatment and developed an 8-week, 8-drug induction/consolidation regimen (protocol I) that remains the core of modern ALL therapy. After this 8-week phase, children with ALL received cranial irradiation and therapy quite analogous to contemporary maintenance chemotherapy. Remarkably, this approach led to cure rates of over $50 \%$. Convinced that even more children could be cured, Dr Riehm reasoned that repeating the 8-week induction with a phase that included some new agents and repeated others would improve the outcome by eliminating residual drug-resistant cells. As completely different drugs for treating ALL had not been identified, Dr Riehm substituted dexamethasone for prednisone, doxorubicin for daunorubicin and 6-thioguanine for mercaptopurine in what he called Protocol II (also now termed as delayed intensification by some groups). This approach was a radical departure from previously accepted ALL therapy and was met with significant skepticism. Many felt that this approach would only increase toxicity and would not improve outcome. Dr Riehm was able to convince investigators in three German centers to run a pilot trial of his regimen, which he dubbed 'BFM' for Berlin, Frankfurt and Münster, the three centers running the trial. The regimen proved to be a major success and has been used as a model for therapy in childhood ALL to the present day!

Dr Riehm also discovered that the early response to induction therapy, as measured by reduction in peripheral blood blast cell count following a 7-day course of single-agent prednisone, could divide patients with ALL into two groups with markedly different outcomes. Although the prednisone response has been proven an easily reproducible parameter for in vivo drug resistance, measurement of early response, now performed primarily by flow cytometry or PCR during induction and after consolidation, is a well-established major prognostic factor used in almost all contemporary ALL treatment regimens.

Because the treatment advances he developed were not always readily accepted by other groups working in the field, Dr Riehm spent a great deal of time and energy promoting the concept of 'BFM'-type therapy around the world. To say that he was successful in this endeavor would be a major understatement. Today, BFM-type therapy is used in the United States, Canada, Europe, South America, Australia, New Zealand, the Middle East and Asia. Under the guidance and tutelage of Dr Riehm, an International BFM study group (I-BFM-SG) has 
Table 1 Overview of study groups, recruitment characteristics and patient numbers

\begin{tabular}{|c|c|c|c|c|}
\hline $\begin{array}{l}\text { Study group } \\
\text { AlEOP }\end{array}$ & $\begin{array}{l}\text { Period of enrollment } \\
1982-2000\end{array}$ & $\begin{array}{c}\text { Age group eligible (years) } \\
\leqslant 15^{\mathrm{a}}\end{array}$ & $\begin{array}{c}\text { No. of patients (eligible/evaluable) } \\
4865\end{array}$ & $\begin{array}{c}\text { No. of studies } \\
5\end{array}$ \\
\hline BFM & $1981-2000$ & $<18$ & 6609 & 5 \\
\hline CCG & 1983-2002 & $<21$ & 13298 & 16 \\
\hline COALL & $1982-2003$ & $<18$ & 1967 & 5 \\
\hline $\mathrm{CPH}$ & 1990-2002 & $<18$ & 730 & 2 \\
\hline DCOG & $1984-2004$ & $<18$ & 1734 & 4 \\
\hline DFCl & 1985-2000 & $<18$ & 1457 & 4 \\
\hline INS & $1984-2003$ & $<18$ & 786 & 3 \\
\hline JCCLSG & $1981-1993$ & $<18$ & 1021 & 4 \\
\hline $\mathrm{NOPHO}$ & 1992-2007 & 1 to $<15$ & 2268 & 2 \\
\hline POG & $1984-2001$ & $<22$ & 7393 & 12 \\
\hline SJCRH & 1984-1999 & $\leqslant 18$ & 1011 & 5 \\
\hline TCCSG & 1984-1995 & 1 to $<15$ & 1846 & 4 \\
\hline TPOG & 1997-2007 & $\leqslant 18$ & 1390 & 2 \\
\hline UK-WPCL & 1980-2001 & $\leqslant 15$ & 6516 & 4 \\
\hline
\end{tabular}

${ }^{a}$ The eligible age group was $<18$ years in the trial AIEOP-95.

AlEOP, Associazione Italiana di Ematologia ed Oncologia Pediatrica; BFM, Berlin-Frankfurt-Münster ALL Study Group; CCG, Children's Cancer Group; COALL, Cooperative ALL Study Group; DCOG, Dutch Childhood Oncology Group; DFCl, Dana-Farber Cancer Institute ALL Consortium; INS, Israeli National Studies of childhood ALL; JCCLSG, Japanese Childhood Cancer and Leukemia Study Group; NOPHO, Nordic Society of Pediatric Hematology and Oncology; POG, Pediatric Oncology Group; SJCRH, St Jude Children's Research Hospital; TCCSG, Tokyo Children's Cancer Study Group; TPOG, Taiwan Pediatric Oncology Group; UKALL, UK Medical Research Council Working Party on Childhood Leukaemia.

been formed, with participation of a large number of countries, some with limited resources. An intercontinental consortium from the I-BFM-SG (called ALL-IC BFM) recently completed their first major clinical trial in a large group of countries from Latin America, East Europe and Asia, with results matching those attained by other major study groups. In fact, BFM type of therapy has also recently been adopted by various adult ALL study groups, resulting in significant improvement in cure rates.

It is clear that Dr Riehm is one of the most influential oncologists in the modern era. He deserves special recognition for his pioneering and prodigious accomplishments, and we appreciate his ongoing friendship and support.

M Schrappe ${ }^{1}$, J Nachman ${ }^{2}$, S Hunger $^{3}$, K Schmiegelow $^{4}$, V Conter ${ }^{5}, \mathrm{G}$ Masera ${ }^{5}, \mathrm{R}$ Pieters ${ }^{6}$ and $\mathrm{CH}$ Pui $^{7}$
${ }^{1}$ Department of Pediatrics, University Medical Center Schleswig-Holstein, Campus Kiel, Kiel, Germany; ${ }^{2}$ Department of Pediatric Hematology/Oncology, University of Chicago Children's Hospital, Chicago, IL, USA; ${ }^{3}$ Department of Pediatric Hematology/Oncology/BMT University of Colorado College of Medicine, The Children's Hospital, Aurora, CO, USA:

${ }^{4}$ Department of Pediatrics, the University Hospital, Rigshospitalet, Copenhagen, Denmark;

${ }^{5}$ Department of Pediatrics, University of Milano-Biococca, Monza, Italy;

${ }^{6}$ Department of Pediatric Oncology, Sophia Children's Hospital, Erasmus University Medical Center, Rotterdam, The Netherlands and

${ }^{7}$ St Jude Children's Research Hospital and the University of Tennessee Health Science Center, Memphis, TN, USA E-mail: m.schrappe@pediatrics.uni-kiel.de 